\title{
Identifying neuropsychiatric disorders in the Medicare Current Beneficiary Survey: the benefits of combining health survey and claims data
}

Sophia Miryam Schüssler-Fiorenza Rose ${ }^{1,2^{*}}$ D, Dawei Xie ${ }^{3}$, Joel E. Streim ${ }^{4,5}$, Qiang Pan ${ }^{3}$, Pui L. Kwong ${ }^{3}$ and Margaret G. Stineman ${ }^{6}$

\begin{abstract}
Background: To address the impact of using multiple sources of data in the United States Medicare Current Beneficiary Survey (MCBS) compared to using only one source of data to identify those with neuropsychiatric diagnoses.

Methods: Our data source was the 2010 MCBS with associated Medicare claims files ( $N=14,672$ beneficiaries). The MCBS uses a stratified multistage probability sample design to select a nationally representative sample of Medicare beneficiaries. We excluded those participants in Medicare Health Maintenance Organizations $(n=3894)$ and performed a cross-sectional analysis. We classified neuropsychiatric conditions according to four broad categories: intellectual/developmental disorders, neurological conditions affecting the central nervous system (Neuro-CNS), dementia, and psychiatric conditions. To account for different baseline prevalence differences of the categories we calculated the relative increase in prevalence that occurred from adding information from claims in addition to the absolute increase to allow comparison among categories.

Results: The estimated proportion of the sample with neuropsychiatric disorders increased to 50.0 (both sources) compared to 38.9 (health survey only) and 33.2 (claims only) with an overlap between sources of only $44.1 \%$. Augmenting health survey data with claims led to an increase in estimated percentage of intellectual/developmental disorders, psychiatric disorders, Neuro-CNS disorders and dementia of 1.3, 5.9, 11.5 and 3.8 respectively. In the community sample, the largest relative increases were seen for dementia (147.6\%) and Neuro-CNS disorders (87.4\%). With the exception of dementia, larger relative increases were seen in the facility sample with the greatest being for intellectual/developmental disorders (121.5\%) and Neuro-CNS disorders (93.8 \%).

Conclusions: The magnitude of potentially underestimated sample proportions using health survey only data varied strikingly according to the category of diagnosis and setting. Augmentation of survey data with claims appears essential particularly when attempting to estimate proportion of the sample affected by conditions that cause cognitive impairment which may affect ability to self-report. Augmenting proxy survey data with claims data also appears to be essential when ascertaining proportion of the facility-dwelling sample affected by neuropsychiatric disorders.
\end{abstract}

Keywords: Administrative claims, Health surveys, Dementia, Intellectual disability, Developmental disabilities, Central nervous system diseases, Mental disorders, Medicare Current Beneficiary Survey, MCBS, Residential facilities

\footnotetext{
* Correspondence: cms@alumni.med.upenn.edu

'Spinal Cord Injury Service, Veterans Affairs Palo Alto Health Care System,

3801 Miranda Ave (MC 140), Palo Alto, CA 94304, USA

${ }^{2}$ Department of Neurosurgery, Stanford University, Stanford, California, USA

Full list of author information is available at the end of the article
} 


\section{Background}

The United States Medicare or "Health Insurance for the Aged and Disabled" program provides coverage for almost 44 million Americans ages 65 and older and 9 million Americans with a long-term disability [1]. People with neuropsychiatric impairments comprise a substantial portion of Medicare beneficiaries, but obtaining accurate prevalence estimates can be challenging. Those with cognitive impairment may under-report conditions associated with cognitive impairment due to lack of insight, stigma and other factors. Surveys may only ask about a limited number of conditions. Similarly, administrative data can have low sensitivity [2] and may miss cases. Administrative data, however, are commonly used in health services research because of its availability. Studies involving dementia patients have shown that self-report, administrative data and other sources are complementary; to obtain accurate prevalence estimates one should use multiple sources of data [3]. However, dementia is only one of several health conditions that can account for cognitive and other neuropsychiatric impairments in the Medicare population. In particular, beneficiaries with intellectual disabilities and those with severe mental illness or dementia comprise a substantial portion of the under-65 disabled Medicare population (37\%) [4]. Since on average they are much younger (62 and $50 \%$ of those with intellectual disability and severe mental illness are under age 45), they tend to be on Medicare longer and can incur substantial Medicare expenditures [4].

There is increasing recognition of the need to improve public health surveillance of people with intellectual and developmental disabilities (ID/DD), with calls for improving data systems and sources from the US Surgeon General in 2002 and 2005 and the Centers for Disease Control and Prevention (CDC) in 2009. One approach to improving public health surveillance is to improve the use of existing data through novel analytic methods [5]. In addition to ID/DD and dementia, other conditions such as severe mental illness, stroke and neurological disorders affecting the brain may lead to cognitive and other neuropsychiatric impairments. These impairments are among the most disabling and persistent. They affect substantial proportions of people who qualify for Social Security Disability Insurance and subsequently for Medicare.

The Medicare Current Beneficiary Survey (MCBS) is an ongoing survey of Medicare beneficiaries which combines multiple sources of data, including Medicare claims and survey data collected from a representative sampling of beneficiaries, to capture the health status, health care use and expenditures of the Medicare population as a whole. The Medicare population differs from the general US population in that it is comprised mainly of people ages 65 and older $84.6 \%$ in 2010) and individuals younger than age 65 with a long term disability (15.4\%). In general, in order to be eligible for Medicare, one must be eligible for either Social Security retirement or disability benefits. A small fraction $(<1 \%)$ of Medicare beneficiaries are eligible because of having end-stage renal disease requiring dialysis or transplant or amyotrophic lateral sclerosis.

The MCBS provides a valuable opportunity to evaluate the benefit of using health survey and claims information to evaluate the prevalence of neuropsychiatric disorders. Although the MCBS is commonly used for cost estimates of the entire population, its unique combination of survey and claims data represents an ideal resource for constructing a method that uses multiple sources of data to identify those with potentially cognitively impairing neuropsychiatric conditions. Although it does not contain a "gold standard" for measuring these conditions, it allows examination of the contributions and potential limitations of each source, and can demonstrate how estimates of the proportion of the population affected by these conditions may vary depending on the data sources used.

The goal of our study was to develop a method that combines various sources of information available in the MCBS to identify people with diagnoses of intellectual/ developmental disabilities, dementia, mental illness and neurological conditions affecting the brain. Since most studies are done either using claims only or survey only, we wished to compare the differences in the prevalence of these disorders using health survey information only, claims only and health survey plus claims information. We performed this comparison in the community sample, long-term care facility sample and overall sample.

\section{Methods}

\section{Medicare Current Beneficiary Survey}

The United States Medicare Current Beneficiary Survey (MCBS) uses a stratified multistage probability sample design to draw a nationally representative sample of Medicare beneficiaries. The first sampling stage selects 107 nationally representative primary sampling units (PSUs) which consist of counties (or multiple counties) with both metropolitan and non-metropolitan areas. The second stage selects zip code clusters within each PSU. The third stage, beneficiaries in the selected zip codes are stratified by seven age groups (under age 45, 45-64, $65-69,70-74,75-79,80-84$, and 85 and older) and then subsampled at rates designed to provide equal probability samples within each of the seven age groups [6]. Younger beneficiaries (under age 65) and the oldest beneficiaries (over age 85) are oversampled to improve estimates for these vulnerable segments of the population [7, 8]. The relative sampling rates can range from a low of 1 (70-74) to a high of almost 4 (under age 45 group) [9]. Every year a new panel is selected, and each panel remains in the 
survey for four years. Participation is not mandatory and initial response rates are about $80 \%$ with follow-up participation rates around $95 \%$.

We used the 2010 Access to Care sample, which consists of Medicare beneficiaries who were enrolled as of January 1, 2010 and remained enrolled the entire year. It includes four panels $(n=14,672)$, the panels entering in 2007, 2008, 2009 and 2010. Their cumulative response rates for the 2010 fall survey were 56.6, 58.9, 61.2 and $77.5 \%$ respectively [10]. The MCBS adjusts its survey weights to account for non-response to reduce potential non response bias [11]. The MCBS divides respondents into the community-dwelling and the long-term care facility sample and tailors the survey so that relevant questions are asked of each sample. The MCBS defines long-term care facilities as facilities with at least three beds which provide "long-term care services throughout the facility or in a separately identifiable unit" [10]. Long-term care facilities include long-term nursing homes, assisted living and retirement communities that provide personal care, psychiatric care facilities, and institutions for persons with intellectual or developmental disorders and adult group homes.

The MCBS fall health status and functioning questionnaire has a community version and a long-term care facility version. The questions and structure of the community and facility surveys are different although the topics are similar. The community survey asks respondents (or their proxy) directly about health diagnoses, using the language, "Has a doctor ever told you [or specified person if asking proxy] that (you/he/she) had [specific diagnosis]?" It also asks which diagnoses were the primary causes of Medicare eligibility for those who qualified for Medicare initially because of a disability rather than age (primarily beneficiaries under age 65). Approximately $10 \%$ of community respondents are interviewed using a proxy. In contrast, no facility respondent is interviewed directly; instead a facility staff member serves as the proxy. Since nursing homes certified by the Centers for Medicare and Medicaid Services are required to perform a clinical assessment of their patients and fill out a Minimum Data Set (MDS) form on each patient, the facility staff member is instructed to refer primarily to a participant's MDS quarterly review and if necessary their full MDS assessment for answers to the MCBS questions. In cases where an MDS assessment is not available, the staff proxy is instructed to refer to the participant's medical chart [10].

For participants in a long-term care facility, the MCBS surveys the facility in which they reside about its characteristics and provides that information in the MCBS files. The MCBS files also contain information from Medicare administrative records for all participants. Medicare claims files of participants are provided with the 2010 Access to Care MCBS files. We limited our analysis to Fee-For-Service (FFS) beneficiaries excluding those who were in an HMO $(n=3894)$ because Medicare HMOs are not required to submit claims. We used all available claim files: inpatient hospital, outpatient hospital, skilled nursing facility, physician/supplier, home health, hospice, and durable medical equipment claims. The Cost and Use dataset contains a file with nursing home MDS assessments of participants in the continuing panels who received an assessment during the year, but not for the entering panel. Information from the MDS file and Medicare claims files can be matched to the Access to Care participants using the participant identifier.

\section{Neuropsychiatric conditions}

The strength of the MCBS is its combination of survey data, administrative data and claims. We wanted to take advantage of these various sources of information to do a broad screen for neurological, cognitive, and psychiatric conditions that may cause or are often associated with cognitive or mental impairment. We classified conditions according to four broad, but clinically distinct categories: intellectual/developmental disorders (ID/ DD), neurological conditions affecting the central nervous system (Neuro-CNS), dementia and psychiatric conditions (Table 1). For each of the 4 broad categories used in building our classification system we developed an approach that captured and combined information from two sources: claims and the MCBS health status and functioning community and facility surveys. Table 1 contains the diagnoses used in each category (column 1), the International Classification of Diseases - 9 (ICD-9) codes used to identify the various diagnoses (column 2), and the health status and functioning survey variables which were used in the community (column 3) and facility (column 4) surveys. ICD-9 codes consist of 3-5 numbers. The first three numbers specify a category, the forth digit specifies the subcategory and the fifth digit specifies the sub-classification. In Table 1 , we use 3 digits when we used all subcategories and subclassifications of a particular category, 4 digits when we used all sub-classifications of a particular subcategory and 5 digits when we only used a particular subclassification.

\section{Intellectual and other developmental disabilities Claims definition}

In addition to ICD-9 codes for intellectual disorder, we chose developmental disorders (and their related ICD-9) codes after a review of the literature relying heavily on the list of conditions associated with intellectual disorder used in the analysis of the 1994/1995 National Health Interview Survey Disability Supplements [12, 13]. We 
Table 1 Medicare Current Beneficiary Survey (MCBS) Variable Names and ICD-9 codes used in classification system

\begin{tabular}{|c|c|c|c|}
\hline $\begin{array}{l}\text { Diagnostic Category } \\
\text { and Sub-Diagnoses }\end{array}$ & ICD-9 Codes & $\begin{array}{l}\text { Community Health } \\
\text { Survey (HS) Variables }\end{array}$ & Facility HS Variables \\
\hline \multicolumn{4}{|c|}{ Intellectual and Developmental Disorders (ID/DD) ${ }^{*}$} \\
\hline Intellectual Disability & $317,318 x, 319$, V792 & ocmental, emcausc $1 /$ emcausc $2=22^{* *}$ & $\mathrm{n} / \mathrm{a}$ \\
\hline Cerebral Palsy & $343 x$ & emcausc $1 /$ emcausc2 $=9$ & cerpalsy \\
\hline Delay/Pervasive DD & 3141, 315xx, 299xx, V93, V98, V99 & none & none \\
\hline Spina Bifida & $741 x$ & none & none \\
\hline Chromosomal & $7580-3,7586-9,7595,7598$ & none & none \\
\hline Brain: Anomalies, Degenerative & $330,740,742,7560,75555$ & none & none \\
\hline Muscular Dystrophy & $3590-2$ & emcausc $1 /$ emcausc2 $=8$ & none \\
\hline Congenital Infections & $7710-2$ & none & none \\
\hline Endocrine, Toxic & $243,2530,7607,33181$ & none & none \\
\hline Metabolic & $270,2772,2775,27781-2,27785-9$ & none & none \\
\hline \multicolumn{4}{|l|}{ Psychiatric Disorders } \\
\hline Schizophrenia/Delusion & 295, 297, 2980, 2984, 2989 & none & schizoph \\
\hline Bipolar & 2960-1, 2964-9 & none & manicdep \\
\hline Depression/Mood & $2962-3,3004,311, v 790$ & ocdeprss, emcausc 1 / emcausc $2=34$ & depress \\
\hline Personality & $301 x x$ & none & none \\
\hline Anxiety/Somatic/Dissociative & $\begin{array}{l}3000 x, 30011-30015,30021-30023 \\
3003-3007,3008 x, 3009,30981\end{array}$ & none & anxiety \\
\hline Eating Disorders & $3071,3075 x$ & none & none \\
\hline ADHD/Conduct/impulse Control & $314 x x, 312 x x$ & none & none \\
\hline Unspecified & $\mathrm{n} / \mathrm{a}$ & ocpsycho, emscausc1/2 = 35 & $\mathrm{n} / \mathrm{a}$ \\
\hline \multicolumn{4}{|l|}{ Neurological (Central Nervous System) } \\
\hline Traumatic Brain Injury & $\begin{array}{l}850-4,8001-4,8006-9,8021-4,8026-9 \\
8031-4,8036-9,8041-4,8046-9 \\
95901,9070,3102,2930,2940\end{array}$ & none & braininj \\
\hline Stroke & $430,431,432 x, 433 x x, 434 x x, 437 x, 438 x x$ & ocstroke, emcausc1/emcausc2 = 16 & stroke \\
\hline Epilepsy & $3450-5,3457-9$ & emcausc1/emcausc2 = 5 & seizure \\
\hline Aphasia & 7843 & none & aphasia \\
\hline Brain: Hydrocephalus, Degenerative & $3313-4,33183,33189,3319,3481$ & none & none \\
\hline Multiple Sclerosis/Demyelinating & $340 x, 3411,3418,3419$ & emcausc1/emcausc2 = 7 & scleros \\
\hline Parkinson/Movement & $332,333.0,333.4$ & ocparkin, emcausc1/emcausc2 = 27 & parknson \\
\hline Brain Cancer & 191, 1920-1, 1983 & occbrain & none \\
\hline \multicolumn{4}{|l|}{ Dementia } \\
\hline Alzheimer's & 3310 & no specific & alzhmr \\
\hline Unspecified & $\begin{array}{l}\text { 290, 2912, 29282, 2941, 2948, 797, } \\
3311,3312,33182\end{array}$ & ocalzhmr, emcausc 1 emcausc2 = 23 & dement \\
\hline
\end{tabular}

"ID/DD Diagnostic Category also used the Minimum Data Set file (ric.mds) variables (ab10a =0, ab5E =1); and the facility interview file (ric7) variable "plactype" = 16 ${ }^{* *}$ emcausc1 and emcausc2 are the variable names which list the diagnosis (coded as a number) that was the cause of Medicare eligibility for people under 65 at the time of their initial MCBS interview. Only one of the two had to list the diagnosis to be included

used ICD-9 codes for the following developmental disorders: intellectual disorder, cerebral palsy, developmental delay, spina bifida (we excluded spina bifida occulta), deformities of brain and skull, chromosomal abnormalities, muscular dystrophy, congenital infections, congenital endocrine disorders such as congenital hypothyroidism and acromegaly, metabolic disorders (Table 1).
Health status and functioning questionnaires

The community health status questionnaire asks "Has a doctor ever told (you/SP) that (you/he/she) had mental retardation?" Those under age 65 were asked an additional question, which provided mental retardation as a possible answer: "Which of these conditions was the cause of (your/SP's) becoming eligible for Medicare?” A 
positive answer to either of these questions was counted as an intellectual disorder. There were no questions specifically asking about other developmental disabilities, but muscular dystrophy and cerebral palsy were among the listed causes of Medicare eligibility and those who had these conditions listed were considered to have a developmental disorder.

The facility health status survey combines ID/DD and mental illness into one question. "Did (SP)'s record indicate any history of mental retardation, mental illness, or developmental disability problems? Exclude diagnoses of organic brain syndrome, Alzheimer's disease, and related dementia." Thus it is not possible to separate out those with an ID/DD purely from the facility health status interview. The survey does contain a specific question on cerebral palsy which was included in the survey definition of ID/DD. Because of this limitation in the MCBS survey, for this category alone, we supplemented the health survey information with information from the MDS file and facility questionnaire. We used the MDS files provided with the Cost and Use data set, which has a question (question ab10a) that allows the identification of ID/DD status. Facilities fill out a survey describing institutional characteristics including type of facility. Participants in a facility that responded that it was an "institution for the mentally retarded/developmentally disabled" were considered to have an ID/DD. The MDS file provided also contains questions on residential history in the prior 5 years (question ab5). We considered participants to have ID/ DD if it was indicated in question ab10a or if they had a history of residential stay in an ID/DD facility.

\section{Psychiatric disorders \\ Claims definition}

Our goal was to include claims that were evidence for a chronic psychiatric disorder [14], thus we excluded codes for acute disorders such as acute psychoses, and adjustment disorders. We also excluded simple phobias. We did not include codes purely related to alcohol and substance abuse disorders. The subcategories of psychiatric disorders included are listed in Table 1.

\section{Health status and functioning questionnaires}

The community health questionnaire asks whether a doctor has ever told a participant that $\mathrm{s} / \mathrm{he}$ has depression or a mental or psychiatric disorder other than depression. Participants in an entering panel who are under age 65 are asked whether depression or a mental disorder other than depression was the cause of Medicare eligibility.

We did not use the facility health status and functioning survey's general question asking about mental disorders combined with mental retardation/developmental disorders. The facility health questionnaire did ask about the following specific psychiatric disorders: anxiety, bipolar disorder, depression, and schizophrenia, which were used in our case definition.

\section{Neurological disorders affecting central nervous system Claims}

For claims we used the ICD-9 codes associated with all of the disorders asked about in the community and facility health surveys (Table 1). In addition we added diagnostic codes for hydrocephalus, encephalopathy and anoxic brain damage. We coded Parkinson's disease using codes for primary (332.1) and secondary (332.2) Parkinson's as well as the code for other degenerative disorders of the basal ganglia (333.0), but not code 333.1 (essential tremor), which has been shown to have low specificity for Parkinson's disease $[15,16]$. We also used the code for Huntington's disease (333.4).

\section{Health status and functioning questionnaires}

The community health status and functioning questionnaire surveys participants about the diagnoses of stroke, Parkinson's and brain cancer (including metastases). In addition to stroke and Parkinson's disease, whether people were eligible for Medicare because of seizure disorders or multiple sclerosis is also recorded. The facility questionnaire has staff report on active diagnoses of brain injury, stroke, seizure disorder, aphasia, multiple sclerosis and Parkinson's disease.

\section{Dementia}

\section{Claims definition}

Prior research has shown that ICD-9 codes are not very sensitive in distinguishing between Alzheimer's disease and non-Alzheimer dementia, but do better when dementia as a broad category (including both types) is used [17]. Therefore we combined the diagnostic codes for Alzheimer's disease and other dementias/cognitive impairment into one category [18] (Table 1).

\section{Health status and functioning questionnaires}

The community health status questionnaire does not distinguish between Alzheimer's disease and other dementias, asking "Has a doctor (ever) told (you/SP) that (you/he/she) had Alzheimer's disease or dementia". In contrast, the facility questionnaire directs the facility proxy to mark off active diseases on the MDS assessment and both Alzheimer's disease and dementia (other than Alzheimer's) are listed as options.

\section{Other health conditions}

For the sake of comparison and for further evaluation of the validity of our method, we also included two common non-neuropsychiatric chronic health conditions arthritis and diabetes. Diabetes, a condition that can be asymptomatic, is a condition which we expected to be 
captured well in administrative data given the frequent use of clinical testing. Arthritis, in contrast, is a condition often identified because of subjective pain complaints which may be better captured with survey data. Both are common in older adults.

We used the National Arthritis Data groups ICD-9 codes for arthritis and other rheumatic conditions which have been used in other studies to estimate populationbased prevalence $[19,20]$. The community survey asks about rheumatoid arthritis and non-rheumatoid arthritis, which are also listed in the causes of Medicare eligibility questions. The facility survey asks about arthritis without distinction of type.

For diabetes, we used the following claims codes to identify those with a diabetes-related claim: 250xx, 3620x, 3572,36641 . The community health status and functioning questionnaire asks about diabetes and the subtype, and we did not count those who only reported gestational or pre-diabetes on the survey as having diabetes. The facility survey asks about diabetes and diabetic retinopathy and a positive answer to either of those was considered evidence of diabetes.

\section{Functional status}

Activities of daily living (ADL) limitations were expressed as five partially hierarchical and mutually exclusive stages ranging from no difficulty in any ADLs (Stage 0) to difficulty with all ADLs (Stage IV). The initial validation studies of ADL stages were performed with the Longitudinal Study of Aging II sample [21-23] and were re-derived in the MCBS community sample [24]. Community participants were asked: "Because of a health or physical problem, (do you/participant if proxy interview) have any difficulty [by (yourself/himself/herself) and without special equipment] with the following: bathing or showering, dressing, eating, getting in or out of bed or chairs, walking, and using the toilet." Those who reported having difficulty or who did not do the activity because of a health problem were considered to have difficulty.

The phrasing for facility ADL questions was somewhat different since the facility questions asked about the degree of assistance required for performing each ADL. We assigned those in facilities who were reported to be independent with the ADL without the need of an assistive device as having no difficulty. Those who needed supervision, any level of assistance or did not do the activity were considered to have difficulty.

Questions about the instrumental activities of daily living (IADLs) are similar in structure to the community ADL questions in both community and facility questionnaires. We used the questions about managing money and using the telephone, and assigned difficulty to those who reported difficulty or who did not perform these activities because of a health or physical problem.

\section{Demographic variables}

Demographic covariates including age, sex, race/ethnicity, marital status and education and presence of a living child were obtained primarily from survey data. Where there was missing data we used other sources. For race/ethnicity, we used Medicare administrative data. For income we filled in with income reported in the 2010 Cost and Use files. We did the same for marital status and presence of living child, but we also filled in missing data from household composition, and helper relationship and proxy relationship data. For example if marital status was missing, but person lived with a spouse, received help from a spouse, or the spouse was a proxy, we counted them as married. Administrative data was also used to determine whether they lived in a metropolitan area. For dual eligible status, and participation in an HMO we used variables, which combined Medicare administrative and survey data. Facility living status was determined by whether they received a facility fall 2010 health status and functioning interview and proxy status for those in the community was noted.

\section{Analysis}

Data analysis was performed with $\mathrm{SAS}^{\circ} 9.4$ software (SAS Institute, Inc., Cary, NC, 2013) and we used the survey procedures to account for the complex survey design. We used the MCBS Access to Care crosssectional weights in all analyses. These weights enable the production of estimates from the sample that are generalizable to the Medicare population. They also enable correction for differential selection probabilities, non-response, and post stratification adjustments [11, 25]. The 2010 Access to Care cross-sectional weights enable calculation of weighted estimates which are representative of the continuously enrolled (from Jan 1 to Dec 31, 2010) Medicare population. All percentages presented incorporate the survey weights and are thus weighted percentages.

To assess the effect of adding MCBS survey information we calculated sample proportions based on health survey only, claims data only, and both sources The ID/ DD category also uses information from the facility and MDS files and these were considered part of "survey" data for this diagnostic category alone. We used descriptive statistics to compare demographic and functional characteristics of those identified with a neuropsychiatric disorder using claims and survey and those without. We compared the demographic characteristics of the two groups as part of evaluating construct validity. We expected that the neuropsychiatric group would have a higher proportion of people in the youngest and oldest groups, a higher prevalence of persons reporting low income, ADL and IADL dysfunction, not being married, being dual eligible, living in a facility and using a proxy. 
Diagnosis proportions were calculated for the community, facility and entire (community and facility together) samples. Differences between the proportion of the sample identified using health survey data only, and the proportions using health survey plus claims were calculated in two ways. One was the absolute difference (delta), obtained from subtracting the health survey proportion from the health survey plus claims proportion:

Delta $(\delta)=$ Health Survey \& claims proportion

- health survey only proportion (absolute difference)

The other was the relative percent increase from the health survey only proportion which was calculated as:

Relative $\%$ increase $=$

$(($ Health Survey + Claims proportion $) /($ Survey only proportion $)-1)$ $* 100 \%$

In addition to the impact on estimated proportion of the sample with a diagnosis, we also wished to examine the overlap between cases identified through claims and those identified through health survey. We expected there to be cases identified by claims that were not identified by health surveys because we used major subdiagnoses of each category regardless of whether there was a question about the diagnosis on the community and facility health surveys. To gain a better understanding of the agreement between sources, we chose individual sub-diagnoses of the psychiatric (depression) and Neuro-CNS (stroke, Parkinson's) categories that had a specific question about the diagnosis in the facility health survey, and the community health survey as well as specific diagnostic codes. We used the total number of cases identified using claims plus health survey information and calculated what percentage were identified through claims alone, health survey alone and both.

\section{Results}

The overall estimated prevalence of neuropsychiatric disorders using health survey plus claims was $50.0 \%$. The distribution of demographic and functional characteristics of those with and without neuropsychiatric disorders followed expected patterns (Table 2). A much greater percentage of those with neuropsychiatric disorders $(10.0 \%)$ lived in a long-term care facility compared to $0.9 \%$ of those without such a disorder. A greater proportion of those with neuropsychiatric disorders were under age 65, female, African American, not married, had low income and dual eligible status compared to those without neuropsychiatric disorders. As expected they had a higher prevalence of difficulty with managing money, using the telephone, and more difficulty with ADLs. Community-dwelling participants with neuropsychiatric disorders had higher rates of proxy usage.
Although the health survey proportion (38.9 \%) and the claims only proportion (33.2\%) were not that different (because of incomplete overlap), using both sources of data greatly increased the estimated sample proportion $(50.0 \%)$ which is a $28.6 \%$ relative increase compared to using health survey alone. The magnitude of the increase varied by diagnostic category and setting (Table 3). For example, the estimated prevalence of ID/DD using health survey alone was $2.8 \%$ for the entire sample (community and facility) Adding claims to the health survey information resulted in an estimated prevalence of $4.1 \%$. Because of the relatively low prevalence of ID/DD disorders in the population, while the absolute difference in prevalence was small $(1.3 \%)$, the relative increase was $45.2 \%$. The largest relative increase was seen with Dementia (91.6\%), followed by NeuroCNS disorders (88.2 \%). Similarly, among the individual diagnoses, the greatest relative increase $(71.3 \%)$ was seen for stroke diagnoses. For depression, the claims proportion was less than half that of the health survey proportion and augmenting health survey data with claims, resulted in the smallest relative increase (12.4 \%). In contrast, for diabetes, the claims proportion was higher than the survey proportion (27.4 vs. 20.0) leading to a larger relative increase when augmenting health survey data with claims.

Strikingly the largest relative increases in diagnosis proportion were seen for the facility sample. With the exception of dementia, the relative increases were greater than those seen in the community sample. Intellectual/ developmental disorders had the largest relative increase (121.5 \%) among the four broad neuropsychiatric disorder categories and stroke had the largest relative increase (131.9\%) among the individual neuropsychiatric diagnoses. In contrast to its relatively low relative increase in the community sample ( $25.2 \%)$, the arthritis proportion had the largest relative increase $(249.6 \%)$ in the facility sample (Table 3).

Only $44.1 \%$ of potential neuropsychiatric disorder cases were identified by both claims and health survey (Fig. 1). The lowest overlap was seen in the ID/DD category where only $17.7 \%$ of cases were identified by both and substantially more cases were identified by health survey alone $(51.2 \%)$ than by claims alone $(31.1 \%)$. The highest overlap was seen in diabetes where $58.4 \%$ of cases were identified by both, $33.3 \%$ by claims alone, and only $8.4 \%$ of cases were identified by health survey alone.

\section{Discussion}

Integrating information from various sources in the MCBS data, most notably health status and functioning survey data with administrative claims increased the identification of people with neuropsychiatric disorders. The estimated proportion of neuropsychiatric disorders 
Table 2 Demographic and Functional Characteristics of those with and without neuropsychiatric disorders (determined using claims plus MCBS survey data)

\begin{tabular}{|c|c|c|c|c|}
\hline \multirow[t]{2}{*}{ Characteristic } & & \multirow{2}{*}{$\begin{array}{l}\text { Overall }(n=10,778) \\
\text { raw } n \text { (column wt. \%) }\end{array}$} & \multirow{2}{*}{$\begin{array}{l}\text { Neuropsych Disorder } \\
(n=5866(50 \%) \\
\text { column wt. \% }\end{array}$} & \multirow{2}{*}{$\begin{array}{l}\text { No Neuropsych Disorder } \\
(n=4912(50 \%)) \\
\text { column wt. \% }\end{array}$} \\
\hline & & & & \\
\hline \multirow[t]{3}{*}{$\overline{\text { Age }}$} & $<65$ & $2163(17.6)$ & 27.3 & 7.9 \\
\hline & $65-79$ & $5074(57.2)$ & 44.4 & 70.0 \\
\hline & $80+$ & $3541(25.2)$ & 28.3 & 22.1 \\
\hline \multirow[t]{2}{*}{ Sex } & Female & $5855(54.3)$ & 57.7 & 50.8 \\
\hline & Male & $4923(45.7)$ & 42.3 & 49.2 \\
\hline \multirow[t]{4}{*}{ Race/ Ethnicity } & White $\mathrm{NH}$ & $8453(79.3)$ & 78.5 & 80.0 \\
\hline & Black NH & $1105(9.3)$ & 10.0 & 8.6 \\
\hline & Hispanic & $711(6.4)$ & 6.6 & 6.2 \\
\hline & Other & $509(5.0)$ & 4.9 & 5.2 \\
\hline \multirow[t]{2}{*}{ Marital Status (miss: 5) } & Married & $4791(49.3)$ & 41.3 & 57.3 \\
\hline & Not Married & $5982(50.7)$ & 58.7 & 42.7 \\
\hline \multirow[t]{2}{*}{ Income } & $<25 K$ & $5971(50.4)$ & 59.7 & 41.0 \\
\hline & $\geq 25 \mathrm{~K}$ & 4807 (49.6) & 40.3 & 59.0 \\
\hline \multirow[t]{3}{*}{ High School Graduate } & No & $2795(23.4)$ & 27.0 & 19.7 \\
\hline & Yes & $7814(75.4)$ & 71.1 & 79.7 \\
\hline & Unknown & $169(1.2)$ & 1.9 & 0.5 \\
\hline \multirow[t]{2}{*}{ In Facility } & No & 9895 (94.6) & 90.0 & 99.1 \\
\hline & Yes & $883(5.4)$ & 10.0 & 0.9 \\
\hline \multirow[t]{2}{*}{ Dual Eligible } & No & $8029(79.4)$ & 70.7 & 88.2 \\
\hline & Yes & $2749(20.6)$ & 29.3 & 11.8 \\
\hline \multirow[t]{5}{*}{ Activities of Daily Living Stage (miss: 36 ) } & 0 & $6557(64.3)$ & 51.0 & 77.7 \\
\hline & । & $1727(15.7)$ & 17.8 & 13.5 \\
\hline & $\|$ & $960(8.3)$ & 12.3 & 4.3 \\
\hline & III & $985(8.3)$ & 12.6 & 4.0 \\
\hline & IV & $513(3.4)$ & 6.4 & 0.5 \\
\hline \multirow[t]{2}{*}{ Difficulty Money (miss: 43) } & Yes & $1901(13.6)$ & 24.0 & 3.3 \\
\hline & No & $8834(86.4)$ & 76.0 & 96.7 \\
\hline \multirow[t]{2}{*}{ Difficulty Phone (miss: 42) } & Yes & $1338(10.2)$ & 15.7 & 4.6 \\
\hline & No & $9398(89.8)$ & 84.3 & 95.4 \\
\hline \multicolumn{5}{|l|}{ Community only } \\
\hline \multirow[t]{2}{*}{ Proxy } & No & $8802(90.6)$ & 86.7 & 94.1 \\
\hline & Yes & $1093(9.4)$ & 13.3 & 5.9 \\
\hline
\end{tabular}

Abbreviations: MCBS Medicare Current Beneficiary Survey, wt. weighted, NH non-Hispanic, Neuropsych neuropsychiatric. Source: Authors' analysis using the Medicare Current Beneficiary Survey, 2010

in the sample using all sources of information varied as one would expect with demographic and functional characteristics, providing some evidence for construct validity. When health survey information was augmented with claims data the estimated neuropsychiatric disorder prevalence increased by almost $30 \%$. Among the four individual neuropsychiatric categories, the increase was most notable for respondents with Neuro-CNS disorders followed by intellectual/developmental disorders.
Surprisingly the increases were greatest for those living in facilities, where the staff member proxy relied on the MDS primarily and the medical chart secondarily, raising the question of the adequacy of MDS assessments. We expected more concordance with claims which are also based on the chart. For facilities, the claims proportion was much closer to the combined proportion than the health survey proportion was for all categories and disorders except depression. 
Table 3 Estimated Weighted Proportions of Neuropsychiatric Disorders in the Medicare Population by Data Source and the effect of combining sources

\begin{tabular}{|c|c|c|c|c|c|}
\hline Diagnostic Category or Disorder & Sample & $\begin{array}{l}\text { Health Survey* } \\
\text { Proportion }\end{array}$ & $\begin{array}{l}\text { Claims } \\
\text { Proportion }\end{array}$ & $\begin{array}{l}\text { Health Survey \& } \\
\text { Claims Proportion }\end{array}$ & $\begin{array}{l}\text { delta }^{\text {all-survey }} \text { (n) }^{*} \\
\text { (relative } \% \text { increase) }\end{array}$ \\
\hline \multirow[t]{3}{*}{ Any Neuropsychiatric Disorder } & Community Only & 36.5 & 30.5 & 47.6 & $11.1(30.3 \%)$ \\
\hline & Facility Only & 79.8 & 79.4 & 91.6 & $11.9(14.9 \%)$ \\
\hline & Overall & 38.9 & 33.2 & 50.0 & $11.1(28.6 \%)$ \\
\hline \multicolumn{6}{|l|}{ Neuropsychiatric Diagnoses Categories } \\
\hline \multirow[t]{3}{*}{ Intellectual/ Developmental Disorder } & Community Only & 2.6 & 1.5 & 3.5 & $0.9(34.8 \%)$ \\
\hline & Facility Only & 6.2 & 10.9 & 13.8 & $7.6(121.5 \%)$ \\
\hline & Overall & 2.8 & 2.0 & 4.1 & $1.3(45.2 \%)$ \\
\hline \multirow[t]{3}{*}{ Psychiatric Disorders } & Community Only & 27.4 & 16.9 & 32.8 & $5.4(19.9 \%)$ \\
\hline & Facility Only & 51.1 & 48.2 & 64.4 & $13.3(25.9 \%)$ \\
\hline & Overall & 28.7 & 18.7 & 34.5 & $5.9(20.5 \%)$ \\
\hline \multirow[t]{3}{*}{ NeuroCNS Disorders } & Community Only & 12.2 & 16.0 & 22.8 & $10.7(87.4 \%)$ \\
\hline & Facility Only & 27.5 & 46.4 & 53.4 & $25.8(93.8 \%)$ \\
\hline & Overall & 13.0 & 17.7 & 24.5 & $11.5(88.2 \%)$ \\
\hline \multirow[t]{3}{*}{ Dementia } & Community Only & 2.0 & 4.3 & 5.0 & $3(147.6 \%)$ \\
\hline & Facility Only & 40.4 & 48.0 & 57.7 & $17.2(42.6 \%)$ \\
\hline & Overall & 4.1 & 6.7 & 7.9 & $3.8(91.6 \%)$ \\
\hline \multicolumn{6}{|l|}{ Individual Neuropsychiatric Disorders } \\
\hline \multirow[t]{3}{*}{ Depression } & Community Only & 25.7 & 10.6 & 28.6 & $2.9(11.2 \%)$ \\
\hline & Facility Only & 39.8 & 33.8 & 50.2 & $10.5(26.3 \%)$ \\
\hline & Overall & 26.5 & 11.8 & 29.8 & $3.3(12.4 \%)$ \\
\hline \multirow[t]{3}{*}{ Stroke } & Community Only & 10.6 & 11.0 & 17.7 & $7.1(67.3 \%)$ \\
\hline & Facility Only & 12.3 & 23.5 & 28.4 & $16.2(131.9 \%)$ \\
\hline & Overall & 10.7 & 11.7 & 18.3 & $7.6(71.3 \%)$ \\
\hline \multirow[t]{3}{*}{ Parkinson's Disease } & Community Only & 1.0 & 1.0 & 1.4 & $0.4(37.1 \%)$ \\
\hline & Facility Only & 5.3 & 9.0 & 9.9 & $4.6(87.4 \%)$ \\
\hline & Overall & 1.2 & 1.4 & 1.8 & $0.6(48.9 \%)$ \\
\hline \multicolumn{6}{|l|}{ Non-Neuropsychiatric Disorders } \\
\hline \multirow[t]{3}{*}{ Diabetes } & Community Only & 19.7 & 27.0 & 29.5 & $9.8(49.4 \%)$ \\
\hline & Facility Only & 24.9 & 34.7 & 37.3 & $12.4(49.8 \%)$ \\
\hline & Overall & 20.0 & 27.4 & 29.9 & $9.9(49.4 \%)$ \\
\hline \multirow[t]{3}{*}{ Arthritis } & Community Only & 56.3 & 48.4 & 70.5 & $14.2(25.2 \%)$ \\
\hline & Facility Only & 19.3 & 62.7 & 67.6 & $48.3(249.6 \%)$ \\
\hline & Overall & 54.3 & 49.2 & 70.4 & $16(29.5 \%)$ \\
\hline
\end{tabular}

*For ID/DD and the overall neuropsychiatric categories, the facility "Health Survey" prevalence contains ID/DD cases identified through the MDS (panels 2007, 2008, 2009) and facility interview files (all panels) in addition to those identified in the facility health status and functioning file

**Delta = difference between all sources prevalence and health survey only prevalence; Relative \% is the percent increase in prevalence from adding claims (delta/health survey prevalence)

Delta may not exactly equal difference of presented numbers because of rounding error

Source: Authors' analysis using the Medicare Current Beneficiary Survey, 2010

In addition, even when the health survey only estimated proportion and claims only estimated proportion did not differ greatly from each other, the increases seen when using both sources suggests each source is capturing different groups of people. Although some studies use surveys as the "gold standard" our findings highlight that this can be inappropriate for neuropsychiatric disorders which can impair a person's self-awareness or memory, thus impairing the ability to report [26]. Using survey information alone for these diagnoses is likely to lead to underestimates of the proportion of the population affected. Therefore, particularly for those disorders associated with 


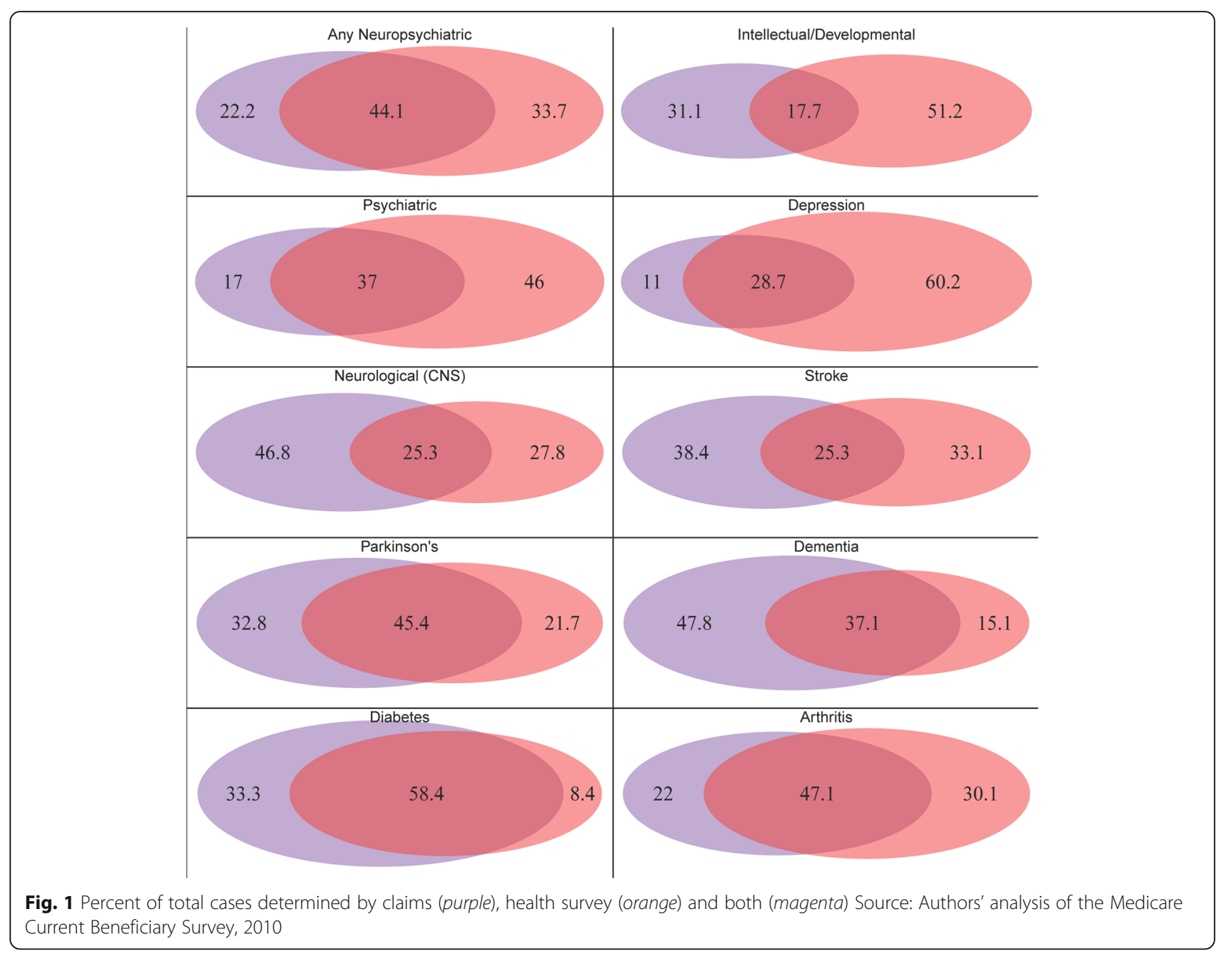

greater cognitive impairments, it appears to be important to augment survey data with claims data. Claims data may also be an important source of augmentation when using interviews done by proxies who may lack full knowledge of the person's health history. For those living in facilities in particular, claims data contributed to a larger relative increase in diagnosis proportion than the increase seen in community-dwellers with the exception of dementia. It may be especially important to augment facility survey data (based on MDS assessments) with claims data.

On the other hand, claims are notorious for lacking sensitivity for identifying certain disorders [27] and are often not appropriate as a sole source of identification. A person with a neuropsychiatric disorder who sees a physician for other reasons may not have this disorder coded in claims. In addition, certain providers may not code for psychiatric disorders if they think they will not be reimbursed for this code and if the visit also covered other disorders for which reimbursement is easier. Typically, claims data are more time-limited than survey data and are not as good as survey data in ascertaining lifetime occurrence of a disorder.
Our study suggests that the adequacy of either claims or survey data varies with the type of disorder and setting. For example, diabetes, an ongoing chronic condition typically followed using objective laboratory measures and often requiring frequent encounters (which logically generate claims), had relatively few cases identified by survey alone. In contrast, conditions like intellectual disability, which may not need new medical interventions or assessment (hence few claims), had a large number of cases identified by survey only. However, more severe forms of intellectual or developmental disorders may impair ability to self-report, so these disorders also had a large percentage identified by claims alone. Thus, heterogeneity among individuals with the same or related diagnostic codes-in their ability to self-report on a survey or in their need for follow-up visits that generate claims-can lead to substantial differences in the sensitivity of survey versus claims data for case ascertainment and prevalence estimation. A similar pattern was seen with stroke. On the one hand, stroke, which may consist of one episode followed by recovery, had a higher percentage of cases identified by 
survey alone than Parkinson's disease, which usually is chronic and requires ongoing health service encounters, making the latter more easily captured through claims. However, one effect of stroke can be anosognosia which would impair ability to report and thus stroke also had a logically higher percentage of cases identified by claims alone with a much smaller overlap between the two sources of data than did Parkinson's. For arthritis there was a large dependence on setting where the survey prevalence was higher in the community, in contrast to the facility setting, where the disorder was better identified by claims.

\section{Limitations}

The major limitation of this study is the absence of a "gold standard" with which to compare our combined measure. Use of self-report as a gold standard, as has been done in some studies [28, 29], is not appropriate for conditions that are commonly associated with impaired cognitive function. Even medical records cannot be considered the "gold standard" as it is mainly a comparison of the agreement of professional coder versus physician/researcher assessment of the medical records; it does not allow one to assess the accuracy of the diagnosis in the medical records [30]. Prospective clinician assessments of the individual could be considered a gold standard but are not practical and economically feasible to perform on a large scale. Without a "gold standard," we are not able to determine our false positive and negative rates. Our findings, however, are reasonably consistent across time in the short term as we obtained consistent results in 2005, 2006 and 2010 data.

In general, claims tend to have much lower sensitivity than specificity $[2,27]$. As such the addition of claims may not completely compensate for under-reporting on surveys. While it is difficult to directly compare our estimated sample diagnosis proportions with the prevalence found in national surveys because of differences in age structure and disorder definition, our estimates for dementia are reasonably close to the estimates reported by a study using the 2002 US population-based Health and Retirement Study Aging, Demographics and Memory Study (HRS-ADAMS) data [31]. The HRS-ADAMs study included careful in home assessment, neurocognitive battery and diagnoses were made by expert multidisciplinary consensus panel with and without reference to the medical records of the participant. For the age groups 70-79, our estimate of $5.6 \%$ is close to the HRS-ADAMs estimate for 71-79 year olds of 4.97 (2.61-7.32). Our overall estimate for the 70 and above age group (11.6 \%) is somewhat lower than the HRSADAMs estimate of 13.93 (11.42-16.44) for the age 71 and above group [31], but still reasonably close considering the different sampling frames, years (2002 vs. 2008) and methodologies. It does not appear that we have excessive false positives for dementia.
For depression our community sample estimate of $28.6 \%$ is substantially higher than that of the National Comorbidity Study-Replication lifetime mood disorder prevalence of $20.8 \%$ [32]. This difference is driven somewhat by the very high rate of depression in our under age 65 sample $(58.7 \%)$ which is consistent with high rates of clinically significant depressive symptoms $(58.3 \%)$ in the under 65 Medicare population reported in other studies [33]. Our over 65 estimate, however, is still higher than the equivalent estimates in other studies and we cannot exclude false positives. The degree to which false positives or false negatives are a concern can depend on the application. We chose to be more inclusive as is appropriate for a broad screen, thus it is likely we included some false positives. Those more concerned about excluding false positives, however, and willing to sacrifice sensitivity, can make the criteria more stringent by requiring multiple occurrences of codes within claims, as has been done in other studies [29,34].

In addition, using broader categories as we have done, can help decrease the number of false negatives (and false positives.) Other studies have shown that the false positive rate is higher when trying to identify specific diagnoses like Alzheimer's disease [13]. Using a more general category such as dementia lowers the false positive rate [13]. Therefore we expect our broader category of neuropsychiatric disorders to have a lower false positive rate than narrower categories.

Finally, the study was performed in a US dataset and a US population-based sample representative of the US Medicare population. Thus, the specific proportions presented and the specifics of the survey data are unlikely to be generalizable to other countries. However the underlying principles of the approach should be able to be applied to other surveys/situations where both types of data are available.

\section{Conclusions}

We have created a broad, four-category screen for distinct neuropsychiatric disorders applicable for population-level studies, which overcomes some of the limitations in using claims data or survey data alone to estimate the proportion of the sample that has been affected by neuropsychiatric disorders. In addition, we have illustrated that it is possible to use a national survey such as the MCBS as a feasible source for surveying adults with developmental disabilities - an understudied population. We provide a detailed methodology to enable others to build on our work as is necessary given updates to ICD codes and survey variables. We illustrate that relying on either claims information alone or self-report alone appears to underestimate the sample proportion of neuropsychiatric disorders. However the magnitude of under-estimation is dependent on the specific category of disorder, the specific 
diagnosis, and the setting. Using both sources together is generally recommended for tasks or projects that require a more accurate estimate of the proportion of the population affected by such conditions. This is especially true of conditions which may impair an individual's ability to self-report (such as dementia), or for which ICD codes have low sensitivity (such as depression). While our study focused on the MCBS, it is likely this approach will also be valuable to augment other surveys querying about neuropsychiatric conditions.

The MCBS provides an ideal opportunity to understand the relationship between self- or close proxy-reported disorders and claims-identified disorders and the potential biases of each source. An understanding of this relationship is invaluable given that it is not financially feasible for most population-level studies to do a full neuropsychiatric assessment. Furthermore, because of its innovative approach to collecting cost data, the MCBS can be used to better evaluate how using only one source of information might bias cost estimates for those conditions, which is of great importance for policy makers. Consequently, both survey and claims data as combined from the MCBS will continue to be useful in US population surveillance and health services research.

\section{Abbreviations}

FFS: Fee-for-Service; HIPAA: Health Insurance Portability and Accountability Act; HMO: Health Maintenance Organization; ICD: International Classification of Diseases; ID/DD: Intellectual/Developmental Disorders; MCBS: Medicare Current Beneficiary Survey; MDS: Minimum Data Set; Neuro-CNS: Neurological disorders affecting the central nervous system

\section{Acknowledgements}

Not applicable.

\section{Funding}

Funding was based on support from the National Institute of Aging of the National Institutes of Health (R01 AG 040105-01A1 (Sean Hennessy, PhD, PharmD) and R01 AG032420-01A1 (Margaret G. Stineman, MD) and by a Postdoctoral Fellowship for Sophia Miryam Schüssler-Fiorenza Rose, MD, PhD (T32-HD-007425) awarded to the University of Pennsylvania by the National Institute of Child Health and Human Development, National Center for Medical Rehabilitation Research. Writing and revising of the manuscript was supported by the Department of Veterans Affairs Office of Academic Affiliations and the Spinal Cord Injury Service of the Veterans Affairs Palo Alto Health Care System Advanced Fellowship Program in Spinal Cord Injury Medicine (Sophia Miryam Schüssler-Fiorenza Rose, MD, PhD). The National Institutes of Health, the Department of Veterans Affairs, and the Centers for Medicare and Medicaid Services (which is only responsible for the initial data) played no role in the design or conduct of the study, in the analysis, interpretation of the data or in the preparation, review, or approval of the manuscript.

\section{Availability of data and materials}

The datasets supporting the conclusions of this article are available from the Centers for Medicare and Medicaid Services (CMS) through the Research Data Assistance Center (ResDAC), http://www.resdac.org/cms-data/file-family/ Medicare-Current-Beneficiary-Survey-MCBS. Once the data request has been reviewed by ResDAC, a formal request package will be sent to CMS by ResDAC.

\section{Authors' contributions}

SMS-FR and MGS conceived and designed the study with input from JES and DX. SMS-FR, QP and PLK analyzed the data. SMS-FR drafted the manuscript. MGS, JES, DX, QP and PLK critically analyzed and edited the manuscript for important intellectual content. All authors approved the final version.

\section{Competing interests}

The authors declare that they have no competing interests.

\section{Consent for publication}

Not applicable.

\section{Ethics approval and consent to participate}

The MCBS is a limited data set which does not contain direct participant identifiers as defined by the United States Health Insurance Portability and Accountability Act of 1996 (HIPAA). A data use agreement was established with the Centers for Medicare and Medicaid Services. The lack of direct identifiers and the data use agreement allows the reuse of MCBS data for research which has the potential to improve the care of Medicare beneficiaries without notification or re-consent of study participants. The institutional review board at the University of Pennsylvania approved the study and the waiver of HIPAA authorization.

\section{Author details}

${ }^{1}$ Spinal Cord Injury Service, Veterans Affairs Palo Alto Health Care System, 3801 Miranda Ave (MC 140), Palo Alto, CA 94304, USA. ²Department of Neurosurgery, Stanford University, Stanford, California, USA. ${ }^{3}$ Department of Biostatistics and Epidemiology, The Center for Clinical Epidemiology and Biostatistics, Perelman School of Medicine, University of Pennsylvania, 423 Guardian Drive, Blockley Hall, Philadelphia, PA 19104-6021, USA. ${ }^{4}$ Geriatric Psychiatry Section, Department of Psychiatry, Perelman School of Medicine, University of Pennsylvania, Philadelphia, Pennsylvania 19104, USA. ${ }^{5}$ VISN 4 Mental IIIness Research Education and Clinical Center, Corporal Michael J. Crescenz Veterans Affairs Medical Center, Philadelphia, Pennsylvania, USA. ${ }^{6}$ Department of Physical Medicine and Rehabilitation, Perelman School of Medicine, University of Pennsylvania, Philadelphia, Pennsylvania 19104, USA.

Received: 1 March 2016 Accepted: 20 September 2016

Published online: 01 October 2016

\section{References}

1. Klees BS, Wolfe CJ, Curtis CA. Brief Summaries Of Medicare \& Medicaid Title XVIII and Title XIX of The Social Security Act. In: Centers for Medicare \& Medicaid Services. 2015.

2. St Germaine-Smith C, Metcalfe A, Pringsheim T, Roberts Jl, Beck CA, Hemmelgarn BR, McChesney J, Quan H, Jette N. Recommendations for optimal ICD codes to study neurologic conditions: a systematic review. Neurology. 2012;79(10):1049-55.

3. Ostbye T, Taylor Jr DH, Clipp EC, Scoyoc LV, Plassman BL. Identification of dementia: agreement among national survey data, medicare claims, and death certificates. Health Serv Res. 2008;43(1 Pt 1):313-26.

4. Foote SM, Hogan C. Disability profile and health care costs of Medicare beneficiaries under age sixty-five. Health Aff. 2001;20(6):242-53.

5. Krahn G, Fox MH, Campbell VA, Ramon I, Jesien G. Developing a health surveillance system for people with intellectual disabilities in the United States. J Policy Pract Intellect Disabil. 2010;7(3):155-66.

6. Petroski J, Ferraro D, Chu A. Ever enrolled medicare population estimates from the MCBS access to care files. Medicare Medicaid Res Rev. 2014;4(2): E1-16.

7. Long B. Health \& Health Care of the Medicare Population: 2010 Appendix A - Technical Documentation for the Medicare Current Beneficiary Survey. Baltimore: Centers for Medicare and Medicaid Services; 2010. p. 184-92.

8. Adler GS, Phil M. Concept and Development of the Medicare Current Beneficiary Survey. In: Proceedings of the Survey Research Methods Section, American Statistical Association. 1998. p. 153-5.

9. Lo A, Chu A. Variance estimation and the components of variance for the Medicare Current Beneficiary Survey Sample. Abstracts of the Section on Survey Methods, American Statistical Association Annual Meeting. 2005: 3333-3342.

10. Centers for Medicare \& Medicaid Services. Medicare Current Beneficiary Survey (MCBS) CY 2006 Public Use File Documentation Introduction. 2006

11. Kautter J, Khatutsky G, Pope GC, Chromy JR, Adler GS. Impact of nonresponse on Medicare Current Beneficiary Survey estimates. Health Care Financ Rev. 2006:27(4):71-93.

12. Tyler Jr CV, Schramm S, Karafa M, Tang AS, Jain A. Electronic health record analysis of the primary care of adults with intellectual and other developmental disabilities. J Policy Pract Intellect Disabil. 2010;7(3):204-10. 
13. Larson SA, Lakin KC, Anderson L, Kwak N, Lee JH, Anderson D. Prevalence of mental retardation and developmental disabilities: estimates from the 1994/ 1995 National Health Interview Survey Disability Supplements. Am J Ment Retard. 2001;106(3):231-52.

14. Owens P, Myers M, Elixhauser A, Brach C. Care of Adults With Mental Health and Substance Abuse Disorders in U.S. Community Hospitals, 2004. Agency for Healthcare Research and Quality, 2007. HCUP Fact Book No. 10. AHRQ Publication No. 07-0008. ISBN 1-58763-229-2. http://archive.ahrq.gov/data/ hcup/factbk10/factbk10.pdf.

15. Swarztrauber $K$, Anau J, Peters D. Identifying and distinguishing cases of parkinsonism and Parkinson's disease using ICD-9 CM codes and pharmacy data. Mov Disord. 2005;20(8):964-70.

16. Louis ED, Applegate LM, Rios E. ICD-9 CM code 333.1 as an identifier of patients with essential tremor: a study of the positive predictive value of this code. Neuroepidemiology. 2007;28(3):181-5.

17. Taylor Jr DH, Ostbye T, Langa KM, Weir D, Plassman BL. The accuracy of Medicare claims as an epidemiological tool: the case of dementia revisited. J Alzheimers Dis. 2009;17(4):807-15.

18. Taylor Jr DH, Fillenbaum GG, Ezell ME. The accuracy of medicare claims data in identifying Alzheimer's disease. J Clin Epidemiol. 2002;55(9):929-37.

19. Centers for Disease Control and Prevention. Arthritis Prevalence and Activity Limitations - United States, 1990. MMWR. 1994:43(24):433-8.

20. Hootman JM, Helmick CG, Schappert SM. Magnitude and characteristics of arthritis and other rheumatic conditions on ambulatory medical care visits, United States, 1997. Arthritis Rheum. 2002;47(6):571-81.

21. Henry-Sanchez JT, Kurichi JE, Xie D, Pan Q, Stineman MG. Do elderly people at more severe activity of daily living limitation stages fall more? Am J Phys Med Rehabil. 2012;91(7):601-10.

22. Stineman MG, Xie D, Pan Q, Kurichi JE, Zhang Z, Saliba D, Henry-Sánchez JT, Streim J. All-cause 1-, 5-, and 10-year mortality in elderly people according to activities of daily living stage. J Am Geriatr Soc. 2012;60(3):485-92.

23. Schüssler-Fiorenza CM, Xie D, Pan Q, Stineman MG. Comparison of complex versus simple activity of daily living staging: validation of simple stages. Arch Phys Med Rehabil. 2013;94(7):1320-7.

24. Stineman MG, Streim JE, Pan Q, Kurichi JE, Schüssler-Fiorenza Rose SM, Xie D. Activity limitation stages empirically derived for Activities of Daily Living $(\mathrm{ADL})$ and instrumental $\mathrm{ADL}$ in the U.S. adult community-dwelling Medicare population. PM R. 2014;6(11):976-87.

25. Briesacher BA, Tjia J, Doubeni CA, Chen Y, Rao SR. Methodological issues in using multiple years of the Medicare current beneficiary survey. Medicare Medicaid Res Rev. 2012;2(1):E1-19.

26. Barrett AM. Rose-colored answers: neuropsychological deficits and patientreported outcomes after stroke. Behav Neurol. 2010;22(1-2):17-23.

27. Wilchesky M, Tamblyn RM, Huang A. Validation of diagnostic codes within medical services claims. J Clin Epidemiol. 2004;57(2):131-41.

28. Noyes K, Liu H, Holloway R, Dick AW. Accuracy of Medicare claims data in identifying parkinsonism cases: Comparison with the Medicare Current Beneficiary Survey. Mov Disord. 2007:22(4):509-14.

29. Hebert PL, Geiss LS, Tierney EF, Engelgau MM, Yawn BP, McBean AM. Identifying persons with diabetes using Medicare claims data. Am J Med Qual. 1999;14(6):270-7

30. O'Malley KJ, Cook KF, Price MD, Wildes KR, Hurdle JF, Ashton CM. Measuring diagnoses: ICD code accuracy. Health Serv Res. 2005;40(5 Pt 2):1620-39.

31. Plassman BL, Langa KM, Fisher GG, Heeringa SG, Weir DR, Ofstedal MB Burke JR, Hurd MD, Potter GG, Rodgers WL, et al. Prevalence of dementia in the United States: the aging, demographics, and memory study. Neuroepidemiology. 2007;29(1-2):125-32.

32. Kessler RC, Berglund P, Demler O, Jin R, Merikangas KR, Walters EE. Lifetime Prevalence and Age-of-Onset Distributions of DSM-IV Disorders in the National Comorbidity Survey Replication. Arch Gen Psychiatry. 2005;62(6):593-602.

33. Friedman B, Conwell Y, Delavan RR, Wamsley BR, Eggert GM. Depression and suicidal behaviors in Medicare primary care patients under age 65. J Gen Intern Med. 2005;20(5):397-403.

34. Eichler AF, Lamont EB. Utility of administrative claims data for the study of brain metastases: a validation study. J Neurooncol. 2009;95(3):427-31.

\section{Submit your next manuscript to BioMed Central and we will help you at every step:}

- We accept pre-submission inquiries

- Our selector tool helps you to find the most relevant journal

- We provide round the clock customer support

- Convenient online submission

- Thorough peer review

- Inclusion in PubMed and all major indexing services

- Maximum visibility for your research

Submit your manuscript at www.biomedcentral.com/submit
Biomed Central 